\title{
JNM

\section{Gallbladder Volume Indicated for Gallbladder Dysmotility}

TO THE EDITOR: We read with great interest and enthusiasm the article from Colak et $\mathrm{al}^{1}$ regarding the possible diagnostic indication for impaired gallbladder motility by increased gallbladder volume and/or gallbladder wall thickness in patients with nonalcoholic fatty liver disease (NAFLD). In the study, out of 50 patients with NAFLD, 10 (20\%) were identified with steatosis, 22 (44\%) had borderline nonalcoholic steatohepatitis, and 18 (36\%) had definitive nonalcoholic steatohepatitis, in contrast to 38 healthy controls in order to validate a diagnostic panel referenced to bile movement identified under real-time 2-dimensional ultrasonography. Colak et $\mathrm{al}^{1}$ obtained measurements including fasting gallbladder wall thickness (FGWT), fasting gallbladder volume (FGV), and postprandial gallbladder volume. In overall, those 3 measurements are significantly greater in patients with NAFLD than control subjects $(P=0.001, P=0.019$, and $P<0.001$, respectively). Interestingly, there are significant correlations between FGV and presence of NAFLD $(r=0.30)$, also FGV and body mass index $(r=0.36)$; moreover, the linear stepwise regression analyzed body mass index which was an independent predictor of FGV (beta $=0.29, t=2.33$, $P=0.022)$.

In terms of gallbladder size or volume, we agree that the bigger the volume of gallbladder reflects the lower of the motility; therefore, a difference of volume between fasting and postprandial statues could be reasoning gallbladder contractility, ie, the bigger of the difference is the stronger contractility, similarly, the timing of volume change quicker means the stronger of the contractility. Although there are some other issues in the study, such as the sample size and representing area, which need to be further clarified for elucidating the relationship of gallbladder measurements and gallbladder dysmotility, as well NAFLD, however, it is an excellent study encouraging for clinicians and researchers. Use of real-time 2-dimensional ultrasonography to screen gallbladder dysmotility relevant to NAFLD is indeed imperative for currently increasing prevalence of gallstones upon the association with obesity and diabetes. For example, the prevalence of gallstones in the USA is approximately 10-15\%; it was, in China, about 6.29\% in 1989, and 11.1-15.5\% in $2009 .^{2}$ This means that gallstone occurrence is increasing approximately 2 -fold every 10 years. ${ }^{3}$ On the other hand, the changes of size and contractility of the gallbladder is due to the alternation of the intrinsic property of the gallbladder, therefore, the altered gallbladder motility constitutes a primary factor in the pathogenesis of gallstone and other biliary diseases. ${ }^{4,5}$ Gallbladder muscle tone and motility formed up by slow waves and associated contractions, which are similar to slow waves in gastrointestinal smooth muscle cells. Intestinal slow waves are produced by interstitial cells of Cajal (ICC) in the myenteric plexus ${ }^{6}$; similarly, ICC-like cells in gallbladder initiate the spontaneously rhythmic pacemaker potentials that depolarizations smooth muscle in triggering and synchronizing action potentials for bile movement. Some gastrointestinal motility disorders, such as diabetic gastroparesis, slow transit constipation and post-operational gut dysmotilities, ${ }^{7}$ have been attributed to the impaired pacemaker activity of ICC. Whether or not the dysfunctions of biliary system correlated to the architectures of the nerve and ICC-like cells in gallbladder is essentially critical to characterize these type of cells the possible biological and physiological roles, and establish those of FGWT, FGV, and postprandial gallbladder volume as biomarkers for varieties of the gallbladder dysmotilities.

Linlin Liu, ${ }^{1}$ Bing Wu, ${ }^{1}$ and Jianlin $\mathrm{Lv}^{2}$ ${ }^{1}$ Guangxi University of Chinese Medicine School of Nursing Nanning; China; and ${ }^{2}$ The First Affiliated Hospital of Guangxi University of Chinese Medicine, Nanning, China

1. Colak Y, Bozbey G, Erim T, et al. Impaired gallbladder motility and increased gallbladder wall thickness in patients with nonalcoholic fatty liver disease. J Neurogastroenterol Motil 2016;22:470-476.

2. Zhu L, Aili A, Zhang C, Saiding A, Abudureyimu K. Prevalence of and risk factors for gallstones in Uighur and Han Chinese. World J Gastroenterol 2014;20:14942-14949.

3. Min YX, Yu GW. Epidemiologic study of gallstone in Chinese minority 
groups. Zhongguo Chuji Weishengbaojian 2009;23:80-81.

4. Doggrell SA. New targets in and potential treatments for cholesterol gallstone disease. Curr Opin Investig Drugs 2006; 7: 344-348.

5. Shaffer EA. Gallstone disease: epidemiology of gallbladder stone disease. Best Pract Res Clin Gastroenterol 2006;20:981-996.

6. Huizinga JD, Chen JH, Zhu YF. The origin of segmentation motor activity in the intestine. Nat Commun 2014;5:3326.

7. Forster J, Damjanov I, Lin Z, Sarosiek I, Wetzel P, McCallum RW. Absence of the interstitial cells of Cajal in patients with gastroparesis and correlation with clinical findings. J Gastrointest Surg 2005;9:102-108.

\section{Conflicts of interest: None.}

Author contributions: Linlin Liu and Bing Wu are both equal co-first authors, they wrote the draft; and Jianlin Lv as corresponding author made revisions and corrections on the manuscript. 